Supporting Information for

\title{
Controlling the Growth of Au on Icosahedral Seeds of Pd by Manipulating the Reduction Kinetics
}

Tian Lv, ${ }^{\dagger, \ddagger}$ Xuan Yang, ${ }^{\dagger}$ Yiqun Zheng, ${ }^{\S}$ Hongwen Huang, ${ }^{\dagger}$ Lei Zhang, ${ }^{\dagger}$ Jing Tao, ${ }^{\mathbb{I}}$ Likun Pan, ${ }^{\ddagger}$ and Younan $\mathrm{Xia}^{*}, \dagger, \S$

${ }^{\dagger}$ The Wallace H. Coulter Department of Biomedical Engineering, Georgia Institute of Technology and Emory University, Atlanta, Georgia 30332, United States

${ }^{\ddagger}$ Engineering Research Center for Nanophotonics and Advanced Instrument, Ministry of Education, Department of Physics, East China Normal University, Shanghai 200062, People's Republic of China

${ }^{\S}$ School of Chemistry and Biochemistry and School of Chemical and Biomolecular Engineering, Georgia Institute of Technology, Atlanta, Georgia 30332, United States

"I Condensed Matter Physics and Materials Science Department, Brookhaven National Laboratory, Upton, New York 11973, United States

*Correspondence author: younan.xia@ bme.gatech.edu. Phone: 404-385-3209. 


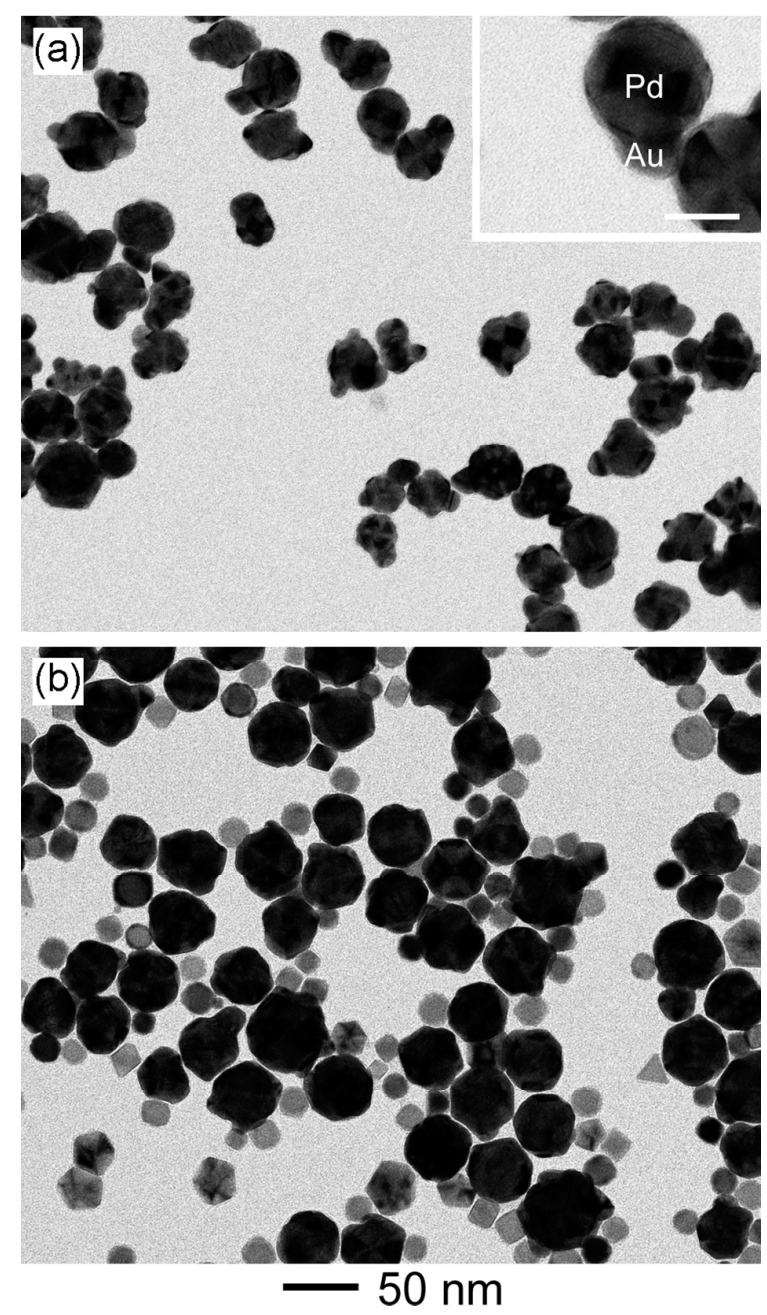

Figure S1. TEM images of nanocrystals obtained by injecting the $\mathrm{HAuCl}_{4}$ in one shot in the presence of AA at two different concentrations: (a) 0.28 and (b) $1.11 \mathrm{mM}$, respectively. The scale bar in the inset of (a) is $20 \mathrm{~nm}$. 


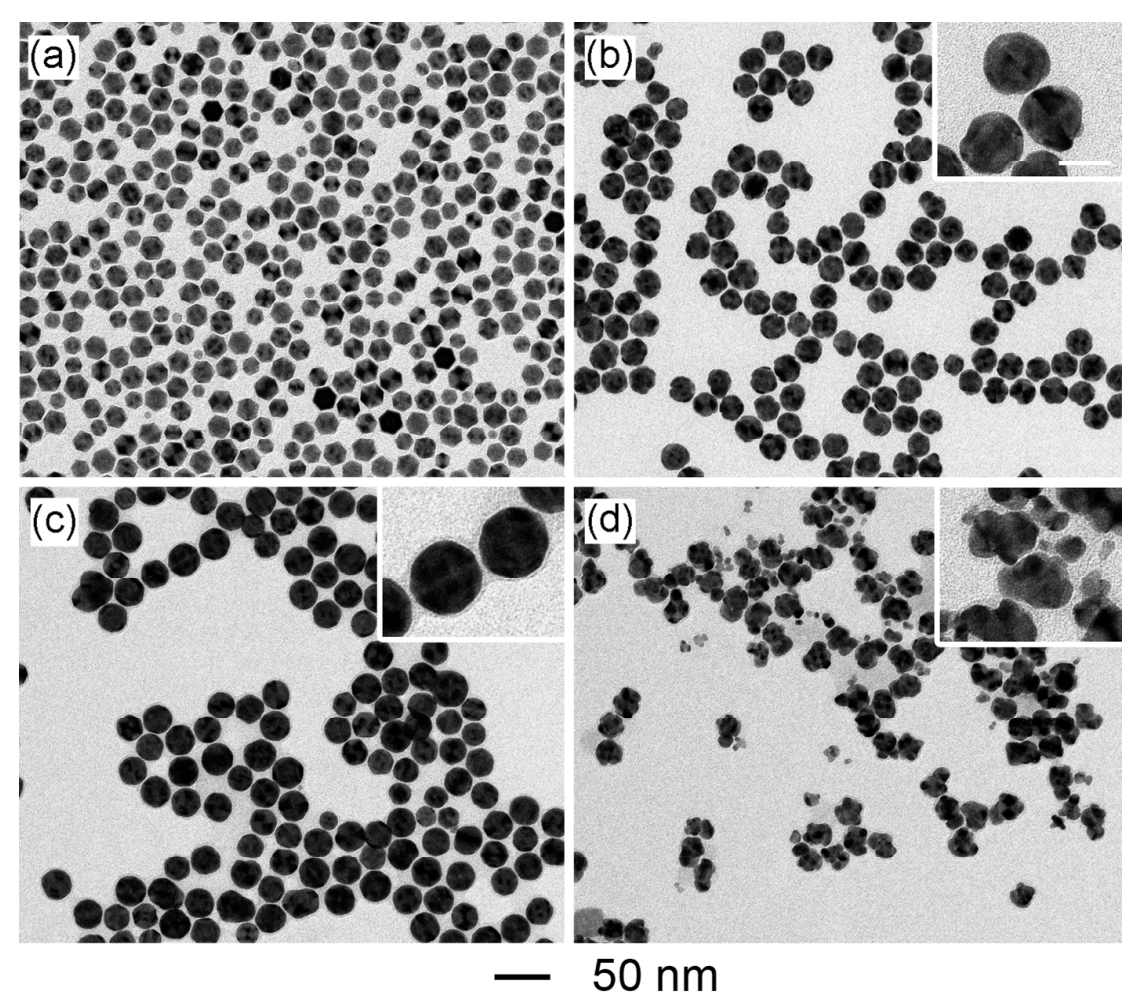

Figure S2. TEM images of (a) the 20-nm Pd icosahedral seeds and (b-d) Pd-Au bimetallic nanocrystals obtained by injecting the $\mathrm{HAuCl}_{4}$ solution at different rates: (b) $0.2 \mathrm{~mL} \mathrm{~h}^{-1}$, (c) $0.5 \mathrm{~mL} \mathrm{~h}^{-1}$, and (d) one shot. The scale bar in the inset of (b) is $20 \mathrm{~nm}$ and applies to all other insets. 